\title{
Decreased number of nymphal stadia in the lygaeid bug, Dimorphopterus japonicus (Heteroptera: Lygaeidae)
}

\author{
Rikiya Sasaki, Tomotaka Hirata, ${ }^{1}$ Fusao Nakasuji* and Kenji Fujisaki ${ }^{2}$ \\ Faculty of Agriculture, Okayama University, Okayama 700-8530, Japan \\ ${ }^{2}$ Graduate School of Agriculture, Kyoto University, Kyoto 606-8502, Japan \\ (Received 16 February 2001; Accepted 15 March 2002)
}

\begin{abstract}
The lygaeid bug, Dimorphopterus japonicus, is a common species in Japan which feeds mainly on the eulalia, Miscanthus sinensis. It shows a marked wing dimorphism of brachyptery and macroptery. Almost all species of the Heteroptera pass through five nymphal stadia, although some exceptions have been noted. In the present study, we observed that D. japonicus had four nymphal stadia before eclosion to adults, while Dimorphopterus pallipes, a closely related species, had five. The number of stadia in D. japonicus did not vary irrespective of sex or wing morph in our observation. This is the first report of a four stadia life cycle in lygaeid bugs. In addition, phytophagous species having a four stadia life cycle are uncommon in the Heteroptera. Although the reasons why D. japonicus has only four nymphal stadia remain unknown, it may have evolved from an ancestral species with a five stadia life cycle like $D$. pallipes.
\end{abstract}

Key words: Lygaeidae, Dimorphopterus japonicus, Dimorphopterus pallipes, nymphal stadium

\section{INTRODUCTION}

The lygaeid bug, Dimorphopterus japonicus, is distributed in the Honshu, Shikoku and Kyushu regions and Tishima Islands in Japan, the Russian Far East and China (Hirashima, 1989). Nymphs and adults feed mainly on the eulalia, Miscanthus sinensis, which is a perennial grass that is widely distributed in Japan. They hide in interstices between the spindle and overlapping young leaves or inside the leaf sheaths of older leaves loosely adhered to the stalk. This bug overwinters in the adult stage in Nagoya, central Japan (Fujita, 1977). It also shows a marked wing dimorphism of brachyptery and macroptery (Fujita, 1977). However, the life history traits of this species have been barely studied.

In Heteroptera, there are ordinarily five nymphal stadia (Slater et al., 1963; Stys and Davidova-Vilimova, 1989; Schuh and Slater, 1995). However, our preliminary observation suggests that nymphs of $D$. japonicus have only four stadia. The closely related species, Dimorphopterus pallipes, which mainly feeds on reeds such as Phragmites australis and Phragmites japonica, also shows a marked wing dimorphism, and goes through five stages (Fujita, 1977; Hirata et al., 1998). The objectives of the present study were to confirm our preliminary observation on the number of stadia and to compare the nymphal growth patterns between $D$. japonicus and $D$. pallipes. Although some exceptions in the number of nymphal stadia have been reported in the true bugs, these are few in number, and those with detailed data are even fewer (Štys and Davidova-Vilimova, 1989). Therefore, the research on basic characters such as the number of nymphal stadia would be not only indispensable for advancing studies of the life history strategy of $D$. japonicus but will also be useful to understanding the biology and evolution of the Heteroptera, which contains many important agricultural pests.

\section{MATERIALS AND METHODS}

Number of nymphal stadia in $D$. japonicus and $D$. pallipes. Laboratory cultures of $D$. japonicus were established with newly emerged adults of 157 females and 170 males collected from an $M$. sinensis patch at Notorohara, the northern district of Okayama Prefecture in western Honshu Island,

\footnotetext{
* To whom correspondence should be addressed at: E-mail: nakasuji@cc.okayama-u.ac.jp

${ }^{1}$ Present address: West Japan Engineering Consultants, INC., Fukuoka 810-0004, Japan
} 
Japan, on August 7, 26 and September 11, 1997. They were reared in groups in 900-ml transparent plastic containers $(9.4 \mathrm{~cm}$ in diameter, $16 \mathrm{~cm}$ in height) under $16 \mathrm{~L} 8 \mathrm{D}$ and $25^{\circ} \mathrm{C}$. Bugs were provided with several chopped stalks and leaves (15 cm long) of $M$. sinensis for food and substrate for oviposition. First-stadium nymphs of $D$. japonicus within $24 \mathrm{~h}$ after hatching were randomly collected from stock cultures in the 3rd generation. This experiment was carried out in the winter season when $M$. sinensis had already wilted in the field, thus nymphs were reared on sugar canes as an alternative food. About 50 individuals were reared on young sugar cane stalks (var. NCo310 cultivated in the greenhouse of Okayama University) placed in a 900-ml plastic container under 16L8D and $25^{\circ} \mathrm{C}$. A total of five containers were used for the experiment. The stalks and containers were replaced with new ones every three days. Ten individuals from one of five containers were sampled at 3-d intervals and were frozen to death. The rearing density in the five containers was adjusted by adding the individuals from other containers set on the same date. The head width was measured using a micrometer under a microscope on the day after each sampling. The wing form of emerged adults was checked for insects in two particular containers.

The 1st-instar stadium of $D$. pallipes were collected randomly within $24 \mathrm{~h}$ after hatching from eggs that had been laid by overwintered female adults collected from a $P$. japonica patch in Kagaminocho, the northern district of Okayama Prefecture, in June 1999. They were confined in 900-ml plastic containers under the same conditions as D. japonicus and provided with the stalks of $P$. japonica. Ten individuals were sampled at 3-d intervals and were frozen to death. The head width was measured using a video micrometer (OLYMPUS, VM-60) on the day after each sampling.

The growth rate index values were calculated as described by Oku (1970). This index measures a ratio of mean head width of a given stadium to that of the previous stadium.

Comparison of nymphal growth between $D$. japonicus and $D$. pallipes. Eggs obtained from cultures in the 2nd generation were used for the experiment. Newly hatched nymphs were individually reared on fresh chopped $M$. sinensis stalks $(6 \mathrm{~cm}$ long) in a $50-\mathrm{ml}$ glass vial $(4 \mathrm{~cm}$ in diameter,

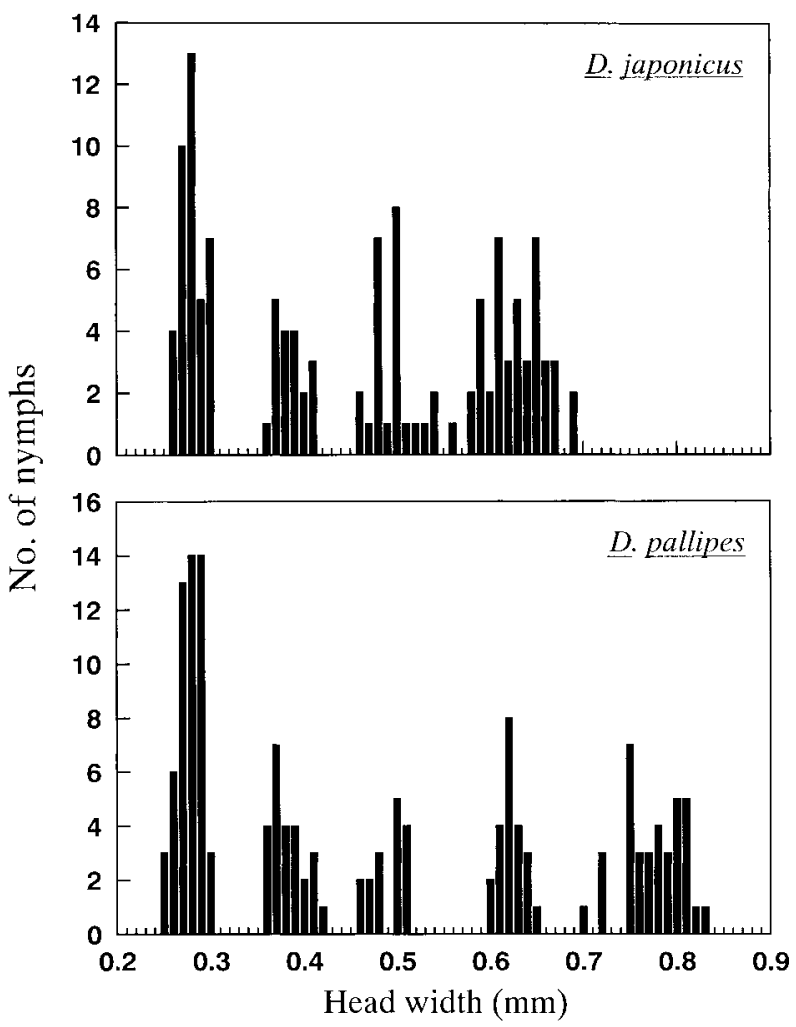

Fig. 1. Frequency distributions of head width of nymphs in D. japonicus and D. pallipes.

$7.5 \mathrm{~cm}$ in height) under $16 \mathrm{~L} 8 \mathrm{D}$ and $25^{\circ} \mathrm{C}$. The number of nymphal stadia was determined by confirming the exuviae daily. Sex and wing form of the individuals were determined after adult eclosion.

As for D. pallipes, newly hatched nymphs from the eggs laid by females collected from a $P$. japonica patch at Noichicho, the southern district of Kochi Prefecture in Shikoku Island, in August 1996, were used for the experiment. They were reared on fresh chopped $P$. japonica stalks $(6 \mathrm{~cm}$ long) in 50-ml glass vials under $16 \mathrm{~L} 8 \mathrm{D}$ and $25^{\circ} \mathrm{C}$ at a density of 5 individuals per container. The number of nymphal stadia was determined by confirming the exuviae daily. Sex and wing form of the individuals were determined after adult eclosion.

\section{RESULTS}

\section{Number of nymphal stadia in D. japonicus and} D. pallipes

Figure 1 shows the frequency distributions of nymphal head width for both species. These figures 
indicate that the nymphal stage is divided into four stadia in D. japonicus and five stadia in D. pallipes.

Table 1 shows the mean head widths at nymphal and adult stages for both species. Each value was based on pooled samples of brachypterous females and brachypterous males from group rearing. The growth pattern up to the 4th stadium was almost the same for the two species. However, in D. pallipes, individuals underwent one more nymphal ecdysis and the adult head width increased further. The growth rate indices tended to decrease as nymphal stadium advanced in both species.

The percentages of macropters among D. japonicus adults (emerged from two containers) were 11.9\% (brachypter: 52, macropter: 7) for females and $8.3 \%$ (brachypter: 44 , macropter: 4 ) for males.

\section{Comparison of nymphal growth between $D$. japonicus and $D$. pallipes}

The durations of the respective nymphal stadia

Table 1. The head width and growth rate index ${ }^{\mathrm{a}}$ at nymphal and adult stages in D. japonicus and D. pallipes

\begin{tabular}{clccc}
\hline Species & Stage & $n$ & $\begin{array}{c}\text { Head width } \\
(\mathrm{mm}) \\
(\bar{x} \pm \mathrm{SE})\end{array}$ & $\begin{array}{c}\text { Growth } \\
\text { rate } \\
\text { index }\end{array}$ \\
\hline D. japonicus & 1st & 39 & $0.278 \pm 0.002$ & - \\
& 2nd & 19 & $0.385 \pm 0.003$ & 1.38 \\
& 3rd & 24 & $0.495 \pm 0.004$ & 1.29 \\
& 4th & 43 & $0.626 \pm 0.004$ & 1.26 \\
& Adult & 34 & $0.702 \pm 0.007$ & 1.11 \\
\hline \multirow{2}{*}{ D. pallipes } & 1st & 53 & $0.277 \pm 0.002$ & - \\
& 2nd & 25 & $0.382 \pm 0.003$ & 1.38 \\
& 3rd & 16 & $0.490 \pm 0.004$ & 1.28 \\
& 4th & 22 & $0.622 \pm 0.003$ & 1.27 \\
& 5th & 36 & $0.775 \pm 0.005$ & 1.24 \\
& Adult & 42 & $0.877 \pm 0.005$ & 1.14 \\
\hline
\end{tabular}

${ }^{\text {a }}$ See text. were compared between D. japonicus and D. pallipes (Table 2). All adults molted into brachypters in $D$. japonicus, while adults of $D$. pallipes emerged as either brachypters or macropters. Daily examination for the exuviae also showed that $D$. japonicus eclosed to adult after four ecdyses. Durations of the first- and final-stadia did not differ between the two species for either sex. However, they differed in middle-stadia. The 2nd-stadium was longer in D. japonicus than in D. pallipes. The 3rdstadium in D. japonicus was longer than 3rd- or 4th-stadium in D. pallipes, and was shorter than the combined 3rd- and 4th-stadia in D. pallipes. Moreover, the change in the duration of each stadium with advanced stadia was slightly different between the two species. In D. japonicus the stadium became increasingly longer from the 2nd- to 4th-stadia, while in D. pallipes each stadium from the 2nd- to the 4th was similar, and the 5th stadium became much longer. The total nymphal period did not differ significantly between two species for either sex ( $p>0.05$, Mann-Whitney $U$-test). The developmental period of females was significantly longer than that of males of both species $(p<0.05$, Mann-Whitney $U$-test).

Figures 2 and 3 show nymphs and a brachypterous female adult of $D$. japonicus and D. pallipes, respectively. First- and 2nd-instars of $D$. pallipes (Fig. 3) were lacking in wing pads. Wing pads appeared first in the 3rd-stadium, where wing pads were very small and did not extend to the 1 st-abdominal segment. In the 4th-stadium, wing pads extended up to the 1st-abdominal segment or beyond. In the 5th-stadium, wing pads exceeded the 2nd-abdominal segment. On the other hand, in $D$. japonicus (Fig. 2) wing pads were absent in the 1 st- and 2nd-instars, and appeared first in 3rd-instars, where they extended to the 1st-abdominal segment. Wing pads in the 4th-stadium of $D$.

Table 2. Comparison of nymphal period between D. japonicus and D. pallipes

\begin{tabular}{|c|c|c|c|c|c|c|c|c|}
\hline \multirow{2}{*}{ Sex } & \multirow{2}{*}{ Species } & \multirow{2}{*}{$n$} & \multicolumn{5}{|c|}{ Stadium (days \pm SE) } & \multirow{2}{*}{ Nymphal stage } \\
\hline & & & $1 \mathrm{st}$ & 2 nd & $3 \mathrm{rd}$ & 4 th & 5 th & \\
\hline \multirow[t]{2}{*}{ Female } & D. japonicus & 22 & $11.1 \pm 0.5$ & $7.8 \pm 0.3$ & $10.1 \pm 0.6$ & $14.0 \pm 0.5$ & - & $43.0 \pm 0.9$ \\
\hline & D. pallipes & 17 & $10.5 \pm 0.7$ & $6.4 \pm 0.5$ & $6.1 \pm 0.4$ & $6.1 \pm 0.4$ & $13.5 \pm 0.6$ & $42.5 \pm 0.9$ \\
\hline \multirow[t]{2}{*}{ Male } & D. japonicus & 26 & $11.2 \pm 0.6$ & $7.5 \pm 0.3$ & $8.5 \pm 0.5$ & $12.3 \pm 0.4$ & - & $39.5 \pm 1.1$ \\
\hline & D. pallipes & 13 & $9.9 \pm 0.9$ & $6.2 \pm 0.5$ & $5.5 \pm 0.2$ & $5.6 \pm 0.5$ & $12.5 \pm 0.7$ & $39.7 \pm 1.2$ \\
\hline
\end{tabular}




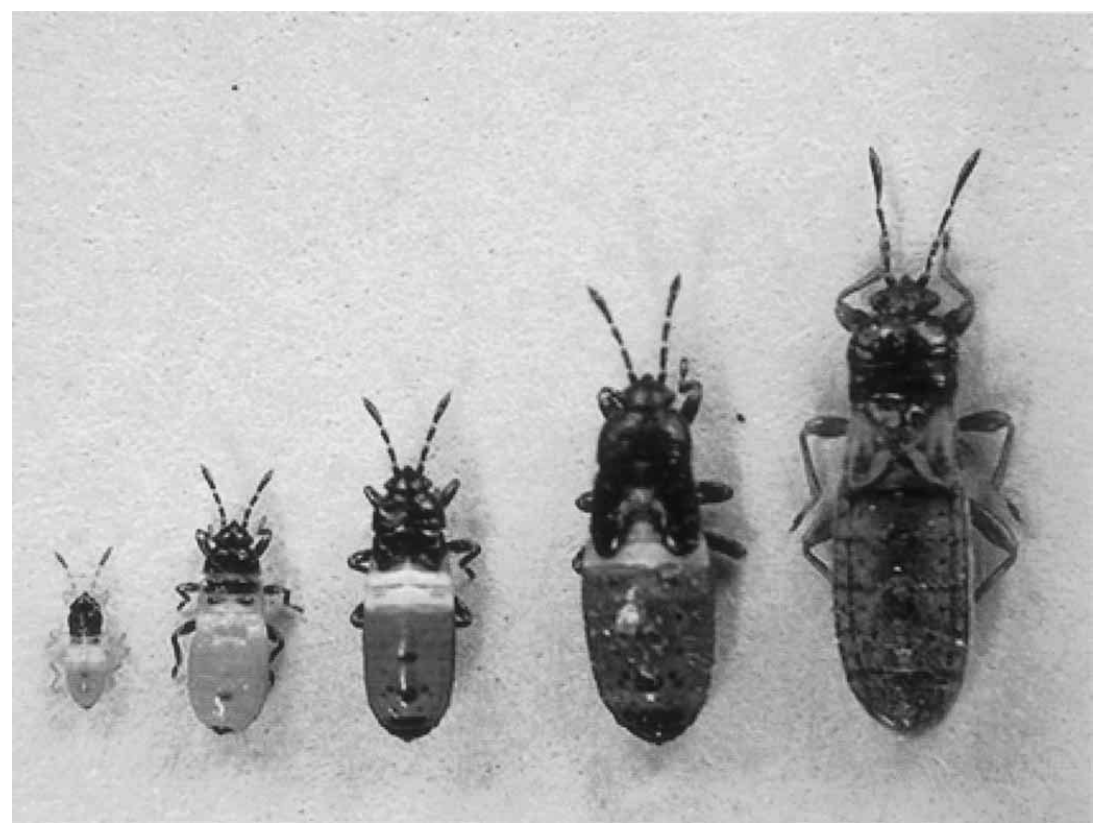

Fig. 2. Nymphs and a female adult of D. japonicus. From the left, the 1st-, 2nd-, 3rd-, and 4th-instars and the brachypterous female (body length: $4.75 \mathrm{~mm}$ ).

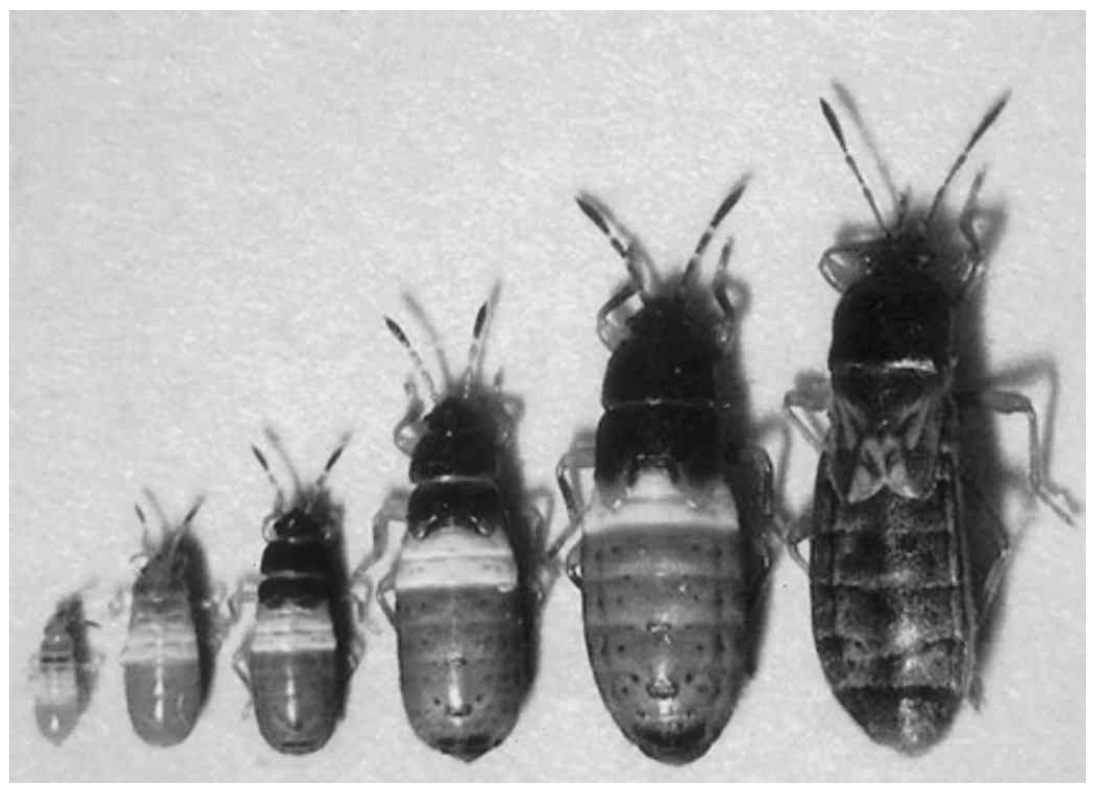

Fig. 3. Nymphs and a female adult of $D$. pallipes. From the left, the 1st-, 2nd-, 3rd-, 4th-, and 5th-instars and the brachypterous female (body length: $6.08 \mathrm{~mm}$ ).

japonicus exceeded the 2nd-abdominal segment similarly to those of the 5th-stadium nymphs of $D$. pallipes.

\section{DISCUSSION}

In general, nymphal development is divided by each ecdyse. The number of immature stages displayed by a given insect is usually typical, within an extremely narrow range of variation, of the species (Beck, 1972). Several cases, specifically in Lepidoptera and Orthoptera, have been reported in which species show a variable number of stadia, depending on genetic, nutritional, or seasonal fac- 
tors. In the cotton bollworm, Helicoverpa armigera, the number of larval stadia ranged from five to seven, depending on the nutritive value of the diet (Casimero et al., 2000). The egg-overwintering ground crickets, Dianemobius nigrofasciatus and Dianemobius mikado, emerge into adults after six nymphal stages under short days (12L12D or 13L11D), but require two or three additional molts during a prolonged nymphal stadia under long days (14L10D or 16L8D) (Masaki and Walker, 1987).

Although almost all species of Heteroptera pass through five nymphal stadia, some exceptions have been noted (Slater et al., 1963; Štys and DavidovaVilimova, 1989; Schuh and Slater, 1995). Such exceptions were reported in Mesovelliidae, Veliidae, Nepidae, Reduviidae, Microphysidae, Miridae, Tingidae, Nabidae, Anthocoridae, Cimicidae, Polyctenidae, Tssaratomidae, and Piesmatidae (Štys and Davidova-Vilimova, 1989). Most were predaceous (except tingids) or parasitic and only two species, Agapophyta bipunctata (Tessaratomidae) and Piesma guadratum (Piesmatidae), were phytophagous. Moreover, only two species, $A$. bipunctata (Tessaratomidae) and $P$. guadratum (Piesmatidae), were likely to have four stadia in Pentatomomorpha.

In Lygaeidae, however, no species with four nymphal stadia have ever been reported (Štys and Davidova-Vilimova, 1989). In the present study, we observed that $D$. japonicus passed through four stadia before eclosion to adults, while D. pallipes, a closely related species, had five (Fig. 1). These results were obtained from both brachypter- and macropter-destined nymphs, because in the experiment where head widths were measured, macropters also emerged (see Results). Therefore, macropters had a four stadia life cycle, too. Moreover, when nymphs were reared under conditions of five individuals in a $50-\mathrm{ml}$ glass vial under $16 \mathrm{~L} 8 \mathrm{D}$ and $28^{\circ} \mathrm{C}$, only one nymph was emerged as a macropterous female after 4 ecdyses (Sasaki, unpublished data). These indicate that the number of stadia four, irrespective of wing form. However, the possibility that this is a characteristic specific to the population used in the present experiment can not be ruled out. In fact, in the predaceous mirid, Cyrtorhinus lividipennis, populations in India and Thailand have five nymphal stages, the Philippines population has four, the Hawaii population has from three to five (Liquido and Nishida, 1985), and the Malaysia population has from four to six (Sivapragasam and Asma, 1985). Therefore, to examine this possibility in D. japonicus, the number of stadia among different geographic populations should be compared in the future.

The reasons why $D$. japonicus has only four nymphal stadia remain unknown. It is likely that $D$. japonicus has evolved from an ancestral species with a five stadia life cycle, such as $D$. pallipes. The results of the present study on wing pad development and the length of each stadium may imply that the 3rd-stadium of $D$. japonicus represents the combinative development of 3rd- and 4th-instars of a 5-stadia species such as D. pallipes. The evolutionary factors that led this species to a four stadia life cycle should be examined in the future.

\section{ACKNOWLEDGEMENTS}

We thank Mr. Tomohiro Harano, Faculty of Agriculture, Okayama University, for his kind assistance in this study. Thanks are also due to Dr. Vic Casimero, Faculty of Agriculture, Okayama University, for his useful comments on the ecdysis of insects.

\section{REFERENCES}

Beck, S. D. (1972) Growth and retrogression in larvae of Trogoderma glabrum (Coleoptera: Dermestidae). 3. Ecdysis and form determination. Ann. Entomol. Soc. Am. 65: 1319-1324.

Casimero, V., R. Tsukuda, F. Nakasuji and K. Fujisaki (2000) Effect of larval diets on the survival and development of larvae in the cotton bollworm, Helicoverpa armigera Hübner (Lepidoptera: Noctuidae). Appl. Entomol. Zool. 35: 69-74.

Fujita, K. (1977) Wing form composition in the field population of the two species of lygaeid bugs, Dimorphopterus pallipes and D. japonicus, and its relation to environmental conditions. Jpn. J. Ecol. 27: 263-267 (in Japanese with English summary).

Hirashima, Y. ed. (1989) A Check List of Japanese Insects, Vol. 1. Kyushu Univ. Entomol. Lab., Fukuoka. 174 pp. (in Japanese).

Hirata, T., F. Nakasuji and K. Fujisaki (1998) Factors determining the wing form of a lygaeid bug, Dimorphopterus pallipes Distant (Heteroptera, Lygaeidae). Chugoku Kontyu 12: 37-41 (in Japanese with English summary).

Liquido, N. J. and T. Nishida (1985) Variation in number of instars, longevity, and fecundity of Cyrtorhinus lividipennis Reuter (Hemiptera: Miridae). Ann. Entomol. Soc. Am. 78: 459-463.

Masaki, S. and T. J. Walker (1987) Cricket life cycles. Evol. Biol. 21: 349-423.

Oku, T. (1970) Studies on life-histories of apple leaf-rollers belonging to the tribe Archipsini. Bull. Hokkaido Agric. Exp. Stn. 19: 15-19 (in Japanese with English summary). 
Schuh, R. T. and J. A. Slater (1995) True Bugs of the World (Hemiptera: Heteroptera) Classification and Natural History. Cornell University Press, Ithaca. $64 \mathrm{pp}$.

Sivapragasam, A. and A. Asma (1985) Development and reproduction of the mirid bug, Cyrtorhinus lividipennis (Heteroptera: Miridae) and its functional response to the brown planthopper. Appl. Entomol. Zool. 20: 373-379.

Slater, J. A., G. S. Tulloch and J. Hanson (1963) The biology and ecology of the Rhyparochrominae of New England (Heteroptera: Lygaeipae). Part I. In Entomologica Americana, Vol. XLIII (New Series). Brooklyn Entomological Society, Lancaster, pp. 67-69.

Štys, P. and J. Davidova-Vilimova (1989) Unusual numbers of instars in Heteroptera: a review. Acta Entomol. Bohemoslov. 86: 1-32. 